\section{Paracetamol como coadyuvante a opioides fuertes en dolor oncológico moderado a intenso: ¿es realmente efectivo?}

\author{
OFELIA LEIVA VÁSQUEZ1 ${ }^{1}$ PEDRO PÉREZ-CRUZ1
}

\section{Paracetamol as an adjuvant to opioids for cancer pain management}

Cancer related pain is one of the most frequent and relevant symptoms in patients with malignant tumors, causing a huge impact in their quality of life. According to the Chilean Public Health System Technical Report of the Cancer Pain Control and Palliative Care Program 2013-2014, 90\% of cancer patients admitted to the Program experienced pain, being moderate or intense in $34 \%$. International and local standards recommend the use of strong opioids (morphine, methadone, or fentanyl) associated with adjuvants such as paracetamol as an initial strategy for pain management. This recommendation assumes that the use of combined analgesics could allow the use of lower opioid doses to obtain similar analgesic effect, decreasing the occurrence of opioid side effects. However, this technical report also describes that there is uncertainty about the impact of paracetamol as an adjuvant in patients with cancer pain who are already receiving strong opioids. This review aims to describe the current state of the art regarding the role of paracetamol as a coadjuvant in cancer pain patients.

(Rev Med Chile 2021; 149: 899-905)

Key words: Acetaminophen; Analgesics, Opioid; Cancer Pain.

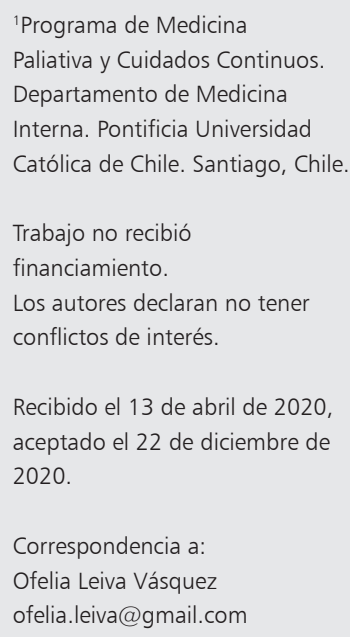

\section{El problema del dolor asociado al cáncer}

$\mathrm{D}$ urante las últimas décadas, la incidencia del cáncer ha aumentado en todo el mundo ${ }^{1}$ y el dolor constituye una de sus complicaciones más frecuentes ${ }^{2}$. En pacientes con cáncer en etapas avanzadas, entre 56 y 75\% de los pacientes experimentan dolor ${ }^{3}$. El dolor no controlado tiene impacto en la funcionalidad de los pacientes, en el ánimo, apetito, sueño y calidad de vida ${ }^{4}$. Afortunadamente, $90 \%$ de los pacientes con dolor por cáncer pueden ser tratados de manera efectiva siguiendo las guías internacionales. En Chile, el cáncer es la segunda causa de muerte ${ }^{5,6}$, y dentro de las garantías establecidas por ley (GES) se encuentra el alivio del dolor por cáncer y cuidados paliativos (ADCCP), para poder responder efectivamente a este grave problema de salud. Reportes del Ministerio de Salud (MINSAL) del programa GES ADCCP señalan que hasta $90 \%$ de los pacientes que ingresan al programa experimentan dolor ${ }^{7}$. En nuestro país, se estima que en el año 2013 ingresaron al menos 26.000 pacientes al programa GES ADCCP en el sistema público, de los cuales 34\% se presentaba con dolor moderado a intenso. Además, a medida que progresa la enfermedad oncológica, la intensidad del dolor también aumenta ${ }^{8}$. Dada la frecuencia e impacto del problema en esta población, ha sido necesario elaborar estrategias que permitan garantizar un alivio efectivo de este síntoma ${ }^{1,7}$. 


\section{Mecanismos fisiopatológicos del dolor asociado al cáncer}

El dolor asociado al cáncer se puede clasificar según sus causas en dolor relacionado a la masa tumoral (casi 80\%), relacionado al tratamiento, o dolor no relacionado al cáncer ${ }^{8}$. Junto con el mecanismo fisiopatológico, existen factores que modulan o modifican la expresión del dolor por parte del paciente (Figura 1). La experiencia del dolor en la que interactúan aspectos físicos, psicosociales y espirituales, se denomina "dolor total", y este concepto fue acuñado por Cicely Saunders, la pionera en el desarrollo de los cuidados paliativos modernos, hace algunas décadas. En esta dirección, se sabe que factores como la presencia de síntomas psicológicos (como depresión o ansiedad), de sufrimiento espiritual, de antecedentes de adicciones o de alteraciones cognitivas, pueden influir en la manera en que el dolor asociado al cáncer se expresa ${ }^{9}$. Dado que la expresión y reporte del dolor asociado al cáncer depende de tanto los mecanismos fisiopatológicos como a factores moduladores, la evaluación del clínico deberá intentar identificar cuáles son los aspectos más importantes que influyen en la experiencia del paciente y elaborar una estrategia terapéutica adecuada.

\section{Evaluación del dolor asociado al cáncer}

Para el adecuado manejo del dolor, es fundamental realizar una evaluación clínica estandarizada que permita identificar la intensidad, los mecanismos fisiopatológicos y los factores moduladores en cada paciente. Para evaluar la intensidad del dolor existen múltiples estrategias. En Chile, la norma técnica GES ADCCP propone varias alternativas estandarizadas, recomendadas por la Organización Mundial de la Salud (OMS) para la pesquisa activa del dolor asociado al cáncer, entre las que destacan la escala verbal análoga (EVERA) y la escala visual análoga $(\mathrm{EVA})^{10}$. La EVERA consiste en que los pacientes asignan un puntaje a la intensidad del dolor en una escala de 0 a 10 . La EVA consiste en una línea horizontal de $10 \mathrm{~cm}$ en un papel en la que sus extremos están delimitados por una marca que expresa "sin dolor" al lado izquierdo y "peor dolor que haya sentido" al lado derecho. Se le pide al paciente que marque una línea vertical cruzando la línea horizontal indicando la intensidad del dolor. Por el reverso, se encuentra la misma línea superpuesta, con una graduación de $1 \mathrm{~cm}$ de ancho, lo que permite identificar la posición en la que se encuentra la línea marcada por el paciente. Esto indica el

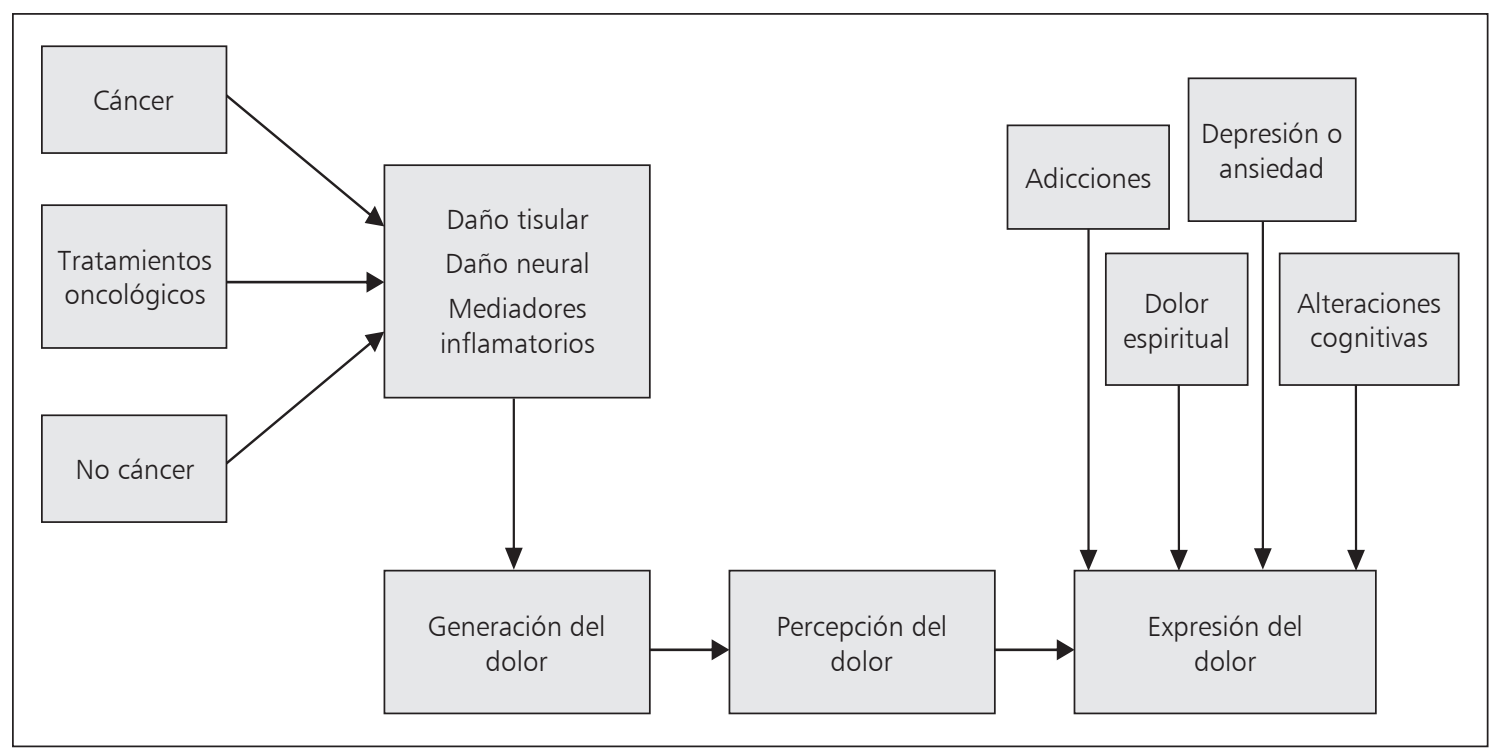

Figura 1. Vía de expresión del dolor (Adaptado de Hui, D \& Bruera, E; Journal of Clinical Oncology, 2014). Dolor total, el dolor se ve afectado por una serie de dominios, que lo que hacen es modular la expresión del dolor. 
puntaje de intensidad del dolor asignado por el paciente en una escala de 0 a 10 . Usando las dos estrategias, según el puntaje, el dolor se clasifica en: ausencia de dolor ( puntaje $=0$ ), dolor leve (puntaje $=1$ a 3 ), dolor moderado (puntaje $=4$ a 6) y dolor intenso (puntaje $=7$ a 10). Se considera que un paciente tiene dolor moderado $y$ que requiere manejo terapéutico intensivo si el paciente tiene un dolor con un puntaje $\geq 4$. Una vez caracterizada la intensidad se progresa con una evaluación clínica estandarizada que incluye entrevista, examen físico e imágenes que permite identificar el mecanismo fisiopatológico. Para la identificación de los factores moduladores se utilizan diferentes instrumentos, algunas de las cuales están descritas en la Tabla 1.

\section{Estrategias para tratar el dolor asociado al cáncer}

Para enfrentar el problema del dolor por cáncer, la OMS presentó en 1986 su estrategia escalonada de analgésicos ${ }^{11,12}$. Esta propuesta de enfoque, que consiste en que el tratamiento analgésico es escalonado y que depende de la intensidad del dolor, se basa más en consensos que en la evidencia. Sin embargo, se ha transformado en un cuidado estándar a nivel internacional y proporciona un marco para el enfoque gradual y sistemático del tratamiento del dolor asociado a cáncer. Por otro lado, su implementación ha sido efectiva en mejorar el control del dolor asociado a cáncer en pacientes oncológicos ${ }^{13}$. Esta estrategia

Tabla 1. Ejemplos de instrumentos para evaluar la presencia de factores moduladores de dolor asociado a cáncer

\begin{tabular}{|c|c|c|}
\hline $\begin{array}{l}\text { Factores } \\
\text { moduladores }\end{array}$ & Instrumentos & Descripción \\
\hline \multirow[t]{3}{*}{ Psicológicos } & $\begin{array}{l}\text { - Hospital anxiety and } \\
\text { depression scale (HADS) }\end{array}$ & $\begin{array}{l}\text { Instrumento de } 14 \text { preguntas con } 2 \text { subescalas de } 7 \text { preguntas cada } \\
\text { una, que pregunta sobre frecuencia de eventos que manifiestan an- } \\
\text { siedad o depresión. Cada pregunta tiene } 4 \text { alternativas que indican } \\
\text { frecuencia o intensidad del evento. La suma de cada una de las } 7 \\
\text { preguntas determina un puntaje para cada escala. Un puntaje de } \geq 8 \\
\text { se considera positivo para escala Ansiedad y/o depresión. }\end{array}$ \\
\hline & $\begin{array}{l}\text { - Edmonton Symptom } \\
\text { Assessment Scale (ESAS) } \\
\text { Ítem depresión }{ }^{10}\end{array}$ & $\begin{array}{l}\text { Instrumento que evalúa la intensidad de } 9 \text { síntomas durante las últimas } \\
24 \text { h en una escala numérica del } 0 \text { al } 10 \text {, y que fue diseñado para utili- } \\
\text { zarse en pacientes con cáncer avanzado. La escala evalúa cada síntoma } \\
\text { de manera independiente. Un puntaje } \geq 4 \text { se considera positivo. }\end{array}$ \\
\hline & - ESAS. Ítem ansiedad ${ }^{10}$ & Ídem \\
\hline Dolor espiritual & $\begin{array}{l}\text { - ESAS-SF. Ítem dolor } \\
\text { espiritual }{ }^{11}\end{array}$ & $\begin{array}{l}\text { Modificación del ESAS que incluye } 2 \text { preguntas que evalúan intensidad } \\
\text { de preocupaciones financieras y de dolor espiritual en una escala del } 0 \\
\text { al } 10 \text {. Un puntaje } \geq 4 \text { se considera positivo. }\end{array}$ \\
\hline $\begin{array}{l}\text { Antecedentes de } \\
\text { adicciones }\end{array}$ & - $\mathrm{CAGE}^{12}$ & $\begin{array}{l}\text { Instrumento que evalúa consumo problemático de alcohol. Se le } \\
\text { pregunta al paciente si ha experimentado alguna de cuatro preguntas } \\
\text { (deseo de disminuir consumo alcohol, le molesta que le pregunten por } \\
\text { el tema, sensación de culpa por consumo y consumo matinal). La pre- } \\
\text { sencia de } 2 \text { preguntas }+ \text {, se considera consumo problemático alcohol. }\end{array}$ \\
\hline $\begin{array}{l}\text { Alteraciones } \\
\text { cognitivas }\end{array}$ & $\begin{array}{l}\text { - Memorial Delirium } \\
\text { Assessment Scale (MDAS) }{ }^{13}\end{array}$ & $\begin{array}{l}\text { Instrumento de } 10 \text { ítems, validado en pacientes con cáncer para la } \\
\text { evaluación del delirium. Puede ser administrado por médico, enfermera } \\
\text { o asistente de investigación sumando un puntaje entre } 0 \text { y } 30 \text { puntos. } \\
\text { Un puntaje mayor a } 13 \text { es sugerente de delirium. }\end{array}$ \\
\hline
\end{tabular}


propone que frente a dolor moderado (peldaño II) o intenso (peldaño III) se debe establecer un esquema analgésico basado en opioides asociado o no a coadyuvantes, entre los que se incluye el paracetamol o los antiinflamatorios no esteroidales (AINES), con opioides débiles para dolor moderado y opioides fuertes para dolor intenso. Un enfrentamiento más actualizado del tratamiento analgésico es el "ascensor analgésico", donde se propone el uso de opioides fuertes (morfina, metadona o fentanyl, entre otros) como analgésico primario en pacientes con dolor moderado o intenso, más coadyuvantes con el fin de lograr un alivio del dolor más rápido y efectivo ${ }^{14}$. En esta guía, además, hacen hincapié de la importancia de los opioides fuertes para el adecuado control del dolor moderado a intenso, como también la necesidad de acceso adecuado ${ }^{15}$.

Este esquema de coadyuvancia se justifica en base a un posible efecto aditivo o sinérgico, dado que los coadyuvantes presentan mecanismos de acción diferentes: Los opioides se unen a receptores opioides y replican los efectos de los opiáceos endógenos, actuando a nivel periférico en la neurona sensitiva y a nivel central en la sinapsis entre la primera y segunda neurona sensitiva, en el asta posterior de la médula espinal, y entre la segunda y tercera neurona sensitiva, a nivel del tálamo, inhibiendo la liberación presináptica y la respuesta postsináptica de neurotransmisores excitatorios. Por otro lado, el paracetamol y los AINES producen analgesia a través de la inhibición de la síntesis de prostaglandinas a nivel central y periférico, disminuyendo la sensibilización de terminaciones nociceptivas mediada prostaglandinas. Esta asociación de opioides y paracetamol o AINES permitiría un mejor control del dolor asociado al cáncer y a disminuir la dosis total de opioides usado al día. Por otro lado, el uso de altas dosis de opioides se asocia a efectos adversos relevantes, tales como, somnolencia, náuseas y vómitos, constipación, entre otros, por lo cual, usar coadyuvantes como el paracetamol o los AINES permitiría reducir sus efectos adversos con la consiguiente optimización de la calidad de vida de los pacientes con dolor asociado a cáncer ${ }^{16}$. Dentro de los coadyuvantes para el tratamiento de dolor asociado a cáncer, si bien los AINES podrían tener un rol analgésico, dada la frecuencia de efectos adversos que tienen, no se recomienda su uso de manera prolongada ni sistemática en pacientes con cáncer. A estas consideraciones se suman la amplia disponibilidad, el relativo bajo costo y el adecuado perfil de seguridad del paracetamol, además de su utilidad demostrada para el manejo del dolor de diversas etiologías, lo que lo hace un medicamento fácil de usar con bajos riesgos ${ }^{17}$.

\section{Efectividad del paracetamol como analgésico para dolor asociado a cáncer}

Estudios internacionales demuestran la efectividad de altas dosis de paracetamol como analgésico, tanto vía oral como intravenosa, en diferentes contextos, particularmente en el dolor postoperatorio ${ }^{18}$. Se sabe que existe una variación en la respuesta a la dosis, con clara evidencia que demuestra que dosis de $1.000 \mathrm{mg}$ vía oral de paracetamol son más efectivas que $500 \mathrm{mg}$ en una gran variedad de escenarios ${ }^{19}$. El paracetamol se encuentra autorizado para ser administrado hasta una dosis de entre $3.000 \mathrm{mg}$ y $4.000 \mathrm{mg}$ al día vía oral, lo que sería seguro para el paciente ${ }^{20}$. La dosis habitual de paracetamol intravenoso para los pacientes de más de $50 \mathrm{~kg}$ es de $650 \mathrm{mg}$ cada cuatro horas o $1.000 \mathrm{mg}$ cada seis horas, sin exceder los $4 \mathrm{~g}$ por día y está contraindicado en paciente con daño hepático crónico grave o falla hepática progresiva. La ventaja del paracetamol intravenoso es que se puede usar en paciente con náuseas y vómitos, anorexia o con incapacidad para ingerir fármacos vía oral por diferentes razones, situaciones frecuentes en pacientes con cáncer. Estudios han comparado la efectividad del paracetamol oral versus paracetamol intravenoso en pacientes quirúrgicos y los resultados han demostrado equivalencia analgésica ${ }^{21} \mathrm{o}$ un leve beneficio hacia el paracetamol intravenoso ${ }^{22}$. En el contexto del cáncer, se sabe que el paracetamol es efectivo en pacientes con dolor leve ${ }^{23}$. Sin embargo, no existe certeza si agregar paracetamol a pacientes con dolor asociado a cáncer moderado a intenso que reciben opioides presenta algún beneficio. Las prácticas internacionales son heterogéneas. En Europa, los pacientes con dolor asociado a cáncer que inician opioides fuertes, se mantienen con paracetamol, mientras que en Norteamérica, el paracetamol se suspende ${ }^{24}$. Los estudios sobre la efectividad del paracetamol como coadyuvante realizados hasta el momento son escasos y la mayoría evalúa pacientes ambulatorios y con dolor crónico estable. En 
un artículo recientemente publicado por nuestro grupo evaluamos, usando la metodología Epistemonikos si la asociación de paracetamol a opioides fuertes tiene algún beneficio para las pacientes, como, por ejemplo, mejor analgesia, reducción en los requerimientos de opioides o disminución de efectos adversos ${ }^{25}$. En este estudio, basado en cinco estudios aleatorizados que incluyeron 171 pacientes, reconocimos que: (a) agregar paracetamol a los opioides fuertes podría hacer poca o nula diferencia en el control del dolor oncológico, con una certeza de la evidencia baja; (b) no está claro si agregar paracetamol a los opioides fuertes tiene algún beneficio sobre los requerimientos analgésicos en pacientes oncológicos, porque la certeza de la evidencia es muy baja; y (c) que no está claro si agregar paracetamol a los opioides fuertes tiene alguna repercusión sobre el bienestar en pacientes oncológicos, porque la certeza de la evidencia es muy baja ${ }^{26-30}$. Otra revisión sistemática publicada en la revista Cochrane concluye que no existe evidencia que el paracetamol oral solo o en combinación con opioides sea beneficioso en los primeros 2 peldaños de la escalera analgésica y no hay claridad del beneficio de este ${ }^{31}$.

\section{Estimación de los costos asociados al uso de paracetamol como analgésico en pacientes con dolor asociado a cáncer moderado a intenso}

En la Guía Clínica GES ADCCP se recomienda usar paracetamol vo en dosis de 500 a $1.000 \mathrm{mg}$ cada 6 a 8 h al día ${ }^{8}$. Siendo conservadores, podríamos estimar que para un adecuado efecto analgésico, los pacientes debieran ingerir dos comprimidos de $500 \mathrm{mg}$ cada $6 \mathrm{~h}$. Si evaluamos el costo de 1 comprimido de paracetamol de $500 \mathrm{mg}$ para el sistema público es de \$7 CLP. Según el Informe Técnico del Programa de ADCCP del año 2014, la estadía promedio de los pacientes en el programa GES es de 122 días. Usando las siguientes suposiciones: Se ingresaron 26.000 pacientes al programa GES ADCCP en el año 2014, de los cuales $34 \%$ tenía dolor moderado a intenso y que estos pacientes usaron una dosis de $4.000 \mathrm{mg}$ de paracetamol al día, y que cada paciente tuvo una estadía media de 122 días, podemos estimar que se usan anualmente $4.313 .920 \mathrm{~g}$ de paracetamol al año. El costo de esta medida es de alrededor de 60,4 millones CLP al año, la que podría ahorrarse si encontramos que esta intervención no es efectiva. Este cálculo conservador subestima el uso de paracetamol en pacientes con dolor asociado a cáncer moderado a intenso, ya que no incluye los pacientes que se encuentran en cuidados paliativos de manera prolongada de años anteriores y que también tiene dolor asociado al cáncer grave. Es importante clarificar que este cálculo de costos asociados al uso de paracetamol no incluye los pacientes con dolor leve que también se podrían beneficiar de esta medida. Tampoco incluye los costos potenciales para el paciente asociado a la carga de ingerir un medicamento frecuentemente, en el contexto donde la ingesta es escasa, y que puede empeorar síntomas como las náuseas y vómitos.

\section{Comentarios finales}

El paracetamol es un fármaco ampliamente usado como analgésico en cáncer. Su eficacia como coadyuvante no es clara en pacientes con dolor por cáncer moderado a intenso y no cuenta con evidencia fuerte que lo sustente. Sin embargo, dado el bajo costo y la facilidad de acceder a este fármaco, es ampliamente utilizado. Hoy también sabemos que el dolor en pacientes con cáncer es frecuente y que en una proporción relevante este dolor es moderado a intenso. Lograr un buen alivio del dolor debe ser una prioridad en los equipos médicos. Por esta razón, es fundamental estudiar mejor el fenómeno del rol del paracetamol en dolor oncológico, de manera de poder implementar de manera oportuna medidas que signifiquen un impacto positivo en el paciente y que los recursos destinados a analgesia estén basados en la mejor evidencia disponible.

Por todo lo anterior, parece no solo necesario estudiar este tema, sino también generar recomendaciones clínicas basadas en la evidencia que nos permitan utilizar los recursos de manera eficiente, beneficiando al paciente en tener un alivio rápido y efectivo del dolor y disminuir la ingesta de fármacos inefectivos.

\section{Referencias}

1. van den Beuken-van Everdingen $\mathrm{MH}$, de Rijke JM, Kessels AG, Schouten HC, van Kleef M, Patijn J. High 
prevalence of pain in patients with cancer in a large population-based study in The Netherlands. Pain. 2007; 132 (3): 312-20.

2. Teunissen SC, Wesker W, Kruitwagen C, de Haes HC, Voest EE, de Graeff A. Symptom prevalence in patients with incurable cancer: a systematic review. J Pain Symptom Manage. 2007; 34 (1): 94-104.

3. Goudas LC, Bloch R, Gialeli-Goudas M, Lau J, Carr DB. The epidemiology of cancer pain. Cancer Invest. 2005; 23 (2): 182-90.

4. Portenoy RK, Ahmed E. Principles of opioid use in cancer pain. J Clin Oncol. 2014; 32 (16): 1662-70.

5. Jiménez de la Jara J, Bastías G, Ferreccio C, Moscoso C, Sagues S, Cid C, et al. A snapshot of cancer in Chile: analytical frameworks for developing a cancer policy. Biological Research. 2015; 48 (10).

6. Instituto Nacional de Estadística. Anuario de Estadísticas Vitales 2015 Santiago, Chile [Available from: http:// www.ine.cl/docs/default-source/publicaciones/2017/ síntesis-de-estadísticas-vitales-2015.pdf?sfvrsn=7.

7. Ministerio de Salud. Informe Técnico Programa Nacional de Alivio del Dolor por Cáncer y Cuidados Paliativos 2013-2014. In: Tumores DMIdCyO, editor. Santiago, Chile2014. https://redcronicas.minsal.cl/wrdprss_minsal/wp-content/uploads/2015/07/PROGRAMA_NACIONAL_ALIVIO_DOLOR2.pdf

8. Ministerio de Salud. Guía Clínica Alivio del Dolor por Cáncer Avanzado y Cuidados Paliativos. Santiago, Chile 2011. https://www.minsal.cl/portal/url/item/72213ed52c2723d1e04001011f011398.pdf.

9. Hui D, Bruera E. A Personalized Approach to Assessing and Managing Pain in Patients With Cancer. J Clin Oncol. 2014; 32 (16): 1640-6.

10. Ministerio de Salud. Norma General Técnica No 32 Programa Nacional de Alivio del Dolor y Cuidados Paliativos. Quinta ed. Santiago, Chile 2009.

11. World Health Organization. Cancer Pain Relief. Geneva, Switzerland: World Health Organization 1986.

12. World Health Organization. WHO's cancer pain ladder for adults 2016. Available from: www.who.int/cancer/ palliative/painladder/en.

13. Jadad AR, Browman GP. The WHO analgesic ladder for cancer pain management. Stepping up the quality of its evaluation. JAMA. 1995; 274 (23): 1870-3.

14. Bandieri E, Romero M, Ripamonti CI, Artioli F, Sichetti D, Fanizza C, et al. Randomized Trial of Low-Dose Morphine Versus Weak Opioids in Moderate Cancer Pain. J Clin Oncol. 2016; 34 (5): 436-42.

15. World Health Organization. WHO Guidelines for the pharmacological and radiotherapeutic management of cancer pain in adults and adolescents 2019 [Available from: https:// www.who.int/ncds/management/palliative-care/cancer-pain-guidelines/en/.

16. Cherny N, Ripamonti C, Pereira J, Davis C, Fallon M, McQuay H, et al. Strategies to manage the adverse effects of oral morphine: An evidence-based report. Journal of Clinical Oncology 2001; 19 (9): 2542-54.

17. Bridger S, Henderson K, Ellis AJ, Glucksman E, Williams R, Henry J. Deaths from low dose paracetamol poisoning - Reply. Brit Med J 1998; 317 (7173): 1656-7.

18. Toms L, McQuay HJ, Derry S, Moore RA. Single dose oral paracetamol (acetaminophen) for postoperative pain in adults. Cochrane Database Syst Rev. 2008(4):CD004602.

19. McQuay HJ, Moore RA. Dose-response in direct comparisons of different doses of aspirin, ibuprofen and paracetamol (acetaminophen) in analgesic studies. $\mathrm{Br} \mathrm{J}$ Clin Pharmacol. 2007; 63 (3): 271-8.

20. Moore RA, Derry S, Wiffen PJ, Straube S, Aldington DJ. Overview review: Comparative efficacy of oral ibuprofen and paracetamol (acetaminophen) across acute and chronic pain conditions. Eur J Pain. 2015; 19 (9): 121323.

21. Politi JR, Davis RL, 2nd, Matrka AK. Randomized Prospective Trial Comparing the Use of Intravenous versus Oral Acetaminophen in Total Joint Arthroplasty. J Arthroplasty. 2017; 32 (4): 1125-7.

22. Hansen RN, Pham AT, Boing EA, Lovelace B, Wan GJ, Miller TE. Comparative analysis of length of stay, hospitalization costs, opioid use, and discharge status among spine surgery patients with postoperative pain management including intravenous versus oral acetaminophen. Curr Med Res Opin. 2017; 33 (5): 943-8.

23. Caraceni A, Hanks G, Kaasa S, Bennett MI, Brunelli C, Cherny $\mathrm{N}$, et al. Use of opioid analgesics in the treatment of cancer pain: evidence-based recommendations from the EAPC. Lancet Oncol. 2012; 13 (2): e58-68.

24. Vardy J, Agar M. Nonopioid drugs in the treatment of cancer pain. J Clin Oncol. 2014; 32 (16): 1677-90.

25. Corsi O, Pérez-Cruz PE. Is it useful to add acetaminophen to high-potency opioids in cancer-related pain? Medwave 2017; 17(Suppl2): e6944 doi: 10.5867/ medwave.2017.6944.

26. Stockler M, Vardy J, Pillai A, Warr D. Acetaminophen (paracetamol) improves pain and well-being in people with advanced cancer already receiving a strong opioid regimen: a randomized, double-blind, placebo-controlled cross-over trial. J Clin Oncol. 2004; 22 (16): 338994.

27. Axelsson B, Borup S. Is there an additive analgesic effect of paracetamol at step 3? A double-blind randomized controlled study. Palliat Med. 2003; 17 (8): 724-5. 
28. Israel FJ, Parker G, Charles M, Reymond L. Lack of benefit from paracetamol (acetaminophen) for palliative cancer patients requiring high-dose strong opioids: a randomized, double-blind, placebo-controlled, crossover trial. J Pain Symptom Manage. 2010; 39 (3): 548-54.

29. Cubero DI, del Giglio A. Early switching from morphine to methadone is not improved by acetaminophen in the analgesia of oncologic patients: a prospective, randomized, double-blind, placebo-controlled study. Support
Care Cancer 2010; 18 (2): 235-42.

30. Tasmacioglu B, Aydinli I, Keskinbora K, Pekel AF, Salihoglu T, Sonsuz A. Effect of intravenous administration of paracetamol on morphine consumption in cancer pain control. Support Care Cancer 2009; 17 (12): 1475 81.

31. Wiffen PJ, Derry S, Moore RA, McNicol ED, Bell RF, Carr DB, et al. Oral paracetamol (acetaminophen) for cancer pain. Cochrane Db Syst Rev. 2017; (7). 